\title{
Ultrafast Protection of SiC MOSFETs With PCB Coil Based Current Sensors
}

This paper was downloaded from TechRxiv (https://www.techrxiv.org).

\section{LICENSE}

CC BY-NC-SA 4.0

SUBMISSION DATE / POSTED DATE

$15-01-2022$ / 24-01-2022

CITATION

Rafiq, Aamir; Pramanick, Sumit (2022): Ultrafast Protection of SiC MOSFETs With PCB Coil Based Current Sensors. TechRxiv. Preprint. https://doi.org/10.36227/techrxiv.18461777.v1

DOI

10.36227/techrxiv.18461777.v1 


\title{
Ultrafast Protection of SiC MOSFETs With PCB Coil Based Current Sensors
}

\author{
Aamir Rafiq, Student Member, IEEE and Sumit Pramanick, Member,IEEE
}

\begin{abstract}
Silicon Carbide (SiC) MOSFETs offer significant advantages in terms of improved efficiency and reduced size of power electronic converters. However, they possess lesser short-circuit withstand time (SCWT) than silicon devices. Consequently, protection circuits for SiC MOSFETs should have a sub-microsecond response time for ensuring device safety. An ultrafast short-circuit protection scheme for TO-247 packaged SiC MOSFETs is presented in this article. The protection scheme utilizes PCB coils to sense the rate of change of current through SiC MOSFETs in a half-bridge circuit. The PCB coil output is integrated to produce a voltage that is proportional to the MOSFET current. The protection circuit compares this voltage with a fault current reference and protects the device when a fault is detected. The design of the protection circuit along with the PCB coil embedded half-bridge circuit board is discussed. Experimental results for a SiC MOSFET subjected to a hard switched fault (HSF) and a fault under load (FUL) are presented, which validates the ultrafast response capability of the protection scheme.
\end{abstract}

Index Terms-Silicon carbide (SiC), short-circuit withstand time (SCWT), current sensor, power converter, finite element method (FEM).

\section{INTRODUCTION}

Silicon Carbide (SiC) MOSFETs offer better performance than silicon IGBTs owing to their excellent material properties. The ability to switch faster, operate at high junction temperature, and withstand high blocking voltage makes these devices suitable for a wide range of applications in electric vehicles and renewable energy technology [1], [2]. However, $\mathrm{SiC}$ MOSFETs possess lesser short-circuit withstand time (SCWT) than Si IGBTs, and consequently, their protection circuits should have lower response time than the state of art techniques for Si IGBTs [3].

SiC MOSFETs with their smaller chip areas possess higher current density than Si IGBTs of similar rating [3], [4]. Small chip area leads to poor thermal capacity, which causes faster junction temperature rise during a short-circuit (SC) event of a SiC MOSFET. Besides smaller chip area, the characteristics of SiC MOSFETs, unlike IGBTs, show a softer saturation of the device current with the increase in device voltage (Fig. 1) owing to short channel effects [5]. Due to this, a large amount of heat is generated within a short duration of time in SiC MOSFETs when compared to Si IGBTs. All these factors limit the SCWT of SiC MOSFETs. SCWT as low as $2 \mu \mathrm{s}$ is reported for the latest generation of $\mathrm{SiC}$ devices [6]. The device failure is either due to the induced thermal runaway or through damage to gate-oxide caused by hot spot formation
[7], [8]. To mitigate such issues, $\mathrm{SC}$ protection circuits for $\mathrm{SiC}$ MOSFETs should have a sub-microsecond response time.

Several protection schemes for SiC MOSFETs are reported in the literature. Protection scheme based on desaturation (DESAT) detection is commonly used with IGBTs [9]. This scheme works by sensing the increase in the collector-emitter voltage $\left(V_{C E}\right)$ when the device current increases and subsequently pulls out of saturation under SC. The threshold for fault detection is at the knee point of the output characteristics, where the IGBT desaturates. During a fault, the current through an IGBT gets limited due to the nature of device characteristics. This current limitation translates to a higher SCWT for IGBTs. Consequently, IGBTs can sustain a longer blanking time with the DESAT-based protection technique. A longer blanking time helps prevent false triggering of the protection circuit due to interference from switching generated $d v / d t$. However, since a large delay cannot be afforded by $\mathrm{SiC}$ MOSFETs due to their low SCWT, a DESAT based protection circuit for SiC MOSFETs with shorter blanking time requires additional design considerations [10], [11].

A DESAT based protection scheme using an auxiliary switch for bypassing $d v / d t$ induced displacement current through the protection circuitry is presented in [5]. Another approach to minimize interference caused by $d v / d t$ switching transient, by employing an FPGA controlled protection circuit, is presented in [12]. The FPGA controls a switch for bypassing the protection circuit in the OFF state of the device, thereby minimizing the influence of switching transients during that duration. Besides delay due to the blanking time, another difficulty with DESAT based protection for SiC MOSFETs is caused by the temperature dependence of the device characteristics, as shown for a commercially available SiC MOSFET in Fig. 1. Consequently, threshold voltage may require external compensation to accommodate temperature dependence of device characteristics [12].

To overcome the limitations associated with DESAT based protection scheme, di/dt signal of the device current can instead be used for fault detection. A fault current evaluation method, using passive integration of the MOSFET $d i / d t$ signal through an RC filter, is presented in [5]. A similar scheme using $d i / d t$ sensing is presented in [14] for implementing both SC protection as well as overload protection for the $\mathrm{SiC}$ MOSFET module. As di/dt detection cannot detect constant load current carried by the switch during conduction, a fixed fault current threshold for both HSF and FUL will result in two separate fault current levels depending on the load current. An RCD-based passive integrator is presented in [15] to mitigate 


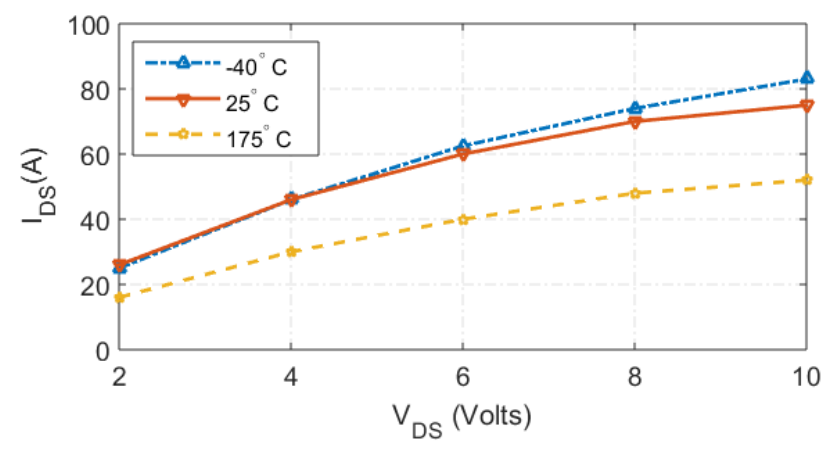

Fig. 1. Output characteristics of a SiC MOSFET (C3M0075120K) at $V_{G S}=15 \mathrm{~V}[13]$.

this issue by preventing discharge of the capacitor voltage and resetting it subsequently. In all these $d i / d t$ based protection schemes $d i / d t$ signal of the device current is sensed through a physical connection from the kelvin source terminal of the device to the gate driver circuit and, therefore, isolation with the power-loop is lost.

Alternatively, device current monitoring through isolated current sensors can instead be used for protecting the device without causing any intrusion inside the power-loop. A current transformer (CT) based protection circuit for a $10 \mathrm{kV} \mathrm{SiC}$ MOSFET module is presented in [16]. The protection circuit compares the measured current from the CT with a fault current reference and withdraws gating pulses to the power device if the device current rises above the fault reference. A similar principle of device protection is employed in [17] for a $\mathrm{SiC}$ module, however, with a Rogowski coil based current sensor. Rogowski coils have earlier been demonstrated for current sensing in press-pack IGBTs in [18]. A comparison of the Rogowski coil based protection method with DESAT detection technique is presented in [19], and superior performance of the current sensing method over DESAT detection based method at different temperatures is demonstrated. It is pertinent to mention that both the current sensing techniques, based on the CT and the Rogowski coil, can measure current with high bandwidth, which is an essential prerequisite for fast response of the protection circuit.

Placing a Rogowski coil [17] or a CT [16] around the terminals of a SiC MOSFET module or an IGBT module is facilitated by the large size of the module package. On the other hand, smaller discrete SiC MOSFET packages do not have sufficient space around their terminals for placing such current sensors. Since the device current gets routed from the device package to the power-loop traces on a PCB, one method to sense the device current is by placing PCB coils to pickup the magnetic field produced by its flow through the power-loop traces [20], [21]. PCB coils are principally similar to Rogowski coils in the sense that they both generate an output voltage that is proportional to the $d i / d t$ of the current which they sense. Consequently, both the coils need an integrator circuit to process the coil output. PCB coils are generally smaller in size than Rogowski coils as they contain lesser turns. However, PCB coils do not have a symmetric distribution of their turns around the current carrying conductor. The small size of PCB coils makes them suitable for integration inside compact layouts designed for discrete devices. Similar to a Rogowski coil, a PCB coil offers the capability to sense the device current with high bandwidth and, therefore, is a suitable choice for implementing an ultrafast protection scheme for discrete SiC MOSFET packages.

This article presents the design of an ultrafast protection scheme for TO-247 packaged SiC MOSFETs with PCB coil based current sensors. While the DESAT based protection scheme suffers from the delay due to blanking time as well as the temperature dependence of device characteristics, the $d i / d t$ based protection scheme relies on a non-isolated kelvin connection on the device package for its implementation. The presented current sensor based protection scheme, with isolation provided through PCB coils, bypasses these limitations while providing an ultrafast response speed.

Two PCB coils are designed for sensing the rate of change of current $(d i / d t)$ through each MOSFET in a half-bridge circuit, fabricated on a four-layered PCB. The design of the half-bridge circuit layout along with the embedded PCB coils is discussed. The PCB coil output is integrated using an analog integrator circuit to produce the current sensor output. Further, the sensor output is compared with a reference fault level using a high-speed comparator for detecting a fault. The frequency response of the PCB coil based current sensor validates its high bandwidth capability, required for providing the protection circuit with an ultrafast response speed. Selection of passive components for the protection circuit is discussed subsequently. Lastly, experimental results are presented, which show a response time of a few nanoseconds for fault clearance with the designed protection circuit, demonstrating an ultrafast speed.

This article is organized as follows. Section II presents the design of the half-bridge circuit board layout featuring embedded PCB coils. Section III presents design of the ultrafast protection circuit. The design of the integrator circuit is discussed, along with the methodology for selecting passive components in the protection circuit. Section IV presents results from the experimental evaluation of the developed protection scheme. The article is concluded in Section V.

\section{PCB Coll Embedded Half-Bridge}

The design of the half-bridge PCB layout with embedded PCB coils is discussed in this section. A PCB coil design on a two-layer double pulse test (DPT) PCB is presented in [22]. In this article, a half-bridge circuit embedded with PCB coils is presented on a four-layered PCB. As will be shown subsequently, the four-layered PCB facilitates smaller separation between the PCB coil and the power-loop traces, which results in higher flux linkage per turn of the PCB coil. This higher flux linkage facilitates the design of the PCB coil with less number of turns, therefore, facilitating a smaller coil size.

Fig. 2(a) shows the schematic of the half-bridge circuit. The schematic layout is shown in Fig. 2(b). The MOSFETs are connected to DC bus bars using two identical traces $T_{1}$ and $T_{2}$. Trace $T_{1}$ connects the positive dc bus $(D C+)$ to the drain 


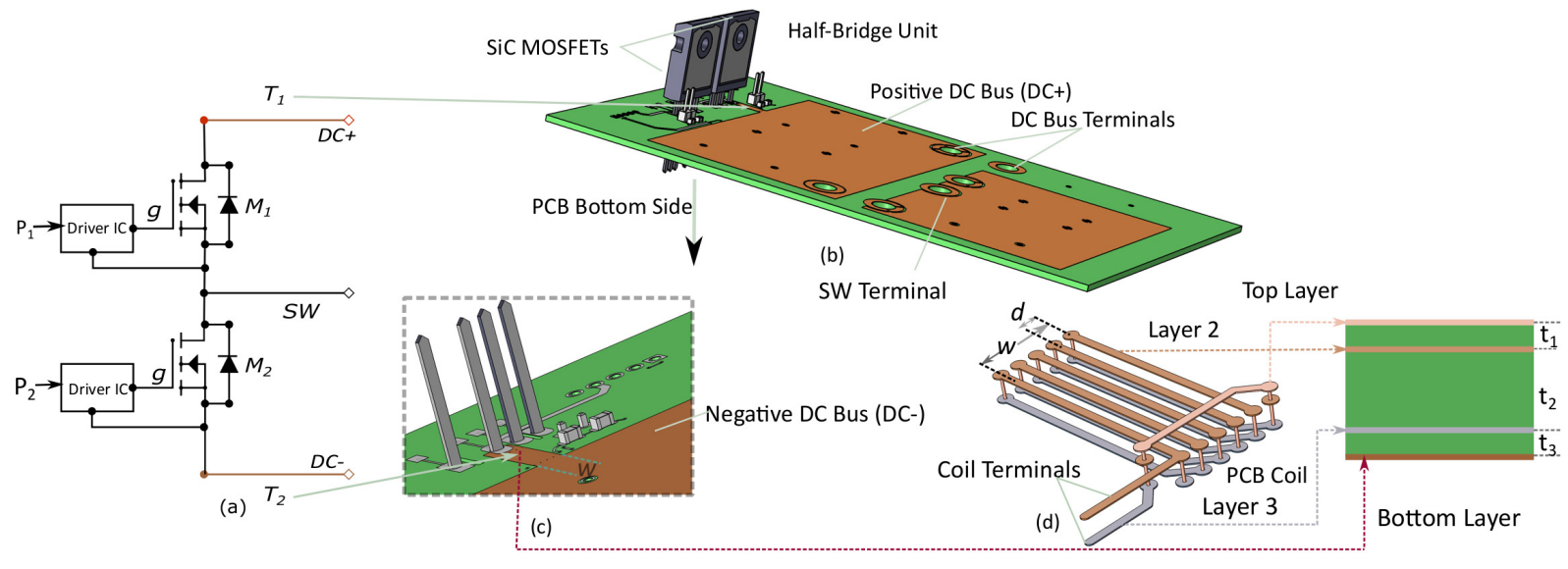

Fig. 2. SiC MOSFET based half-bridge layout featuring embedded PCB coils. (a) Half-bridge schematic. (b) PCB layout. (c) Zoomed in region near the source terminal of MOSFET $M_{2}$ on the bottom layer of PCB. (d) PCB coil implemented above trace $T_{2}$. Also shown is the PCB side view showing the arrangement of coil on the four-layered PCB. The dimensions are $t_{1}=t_{3}=0.35 \mathrm{~mm}, t_{2}=0.7 \mathrm{~mm}, d=0.8 \mathrm{~mm}, \& w=3.2 \mathrm{~mm}$.

of top MOSFET $\left(M_{1}\right)$ on the top-layer and trace $T_{2}$, shown zoomed in Fig. 2(c), connects the source terminal of bottom $\operatorname{MOSFET}\left(M_{2}\right)$ to the negative DC bus $(D C-)$ on the bottomlayer. Traces $T_{1}$ and $T_{2}$ carry the current through MOSFETs, $M_{1}$ and $M_{2}$, respectively. The magnetic field produced by current through these traces is picked up by the embedded PCB coils for sensing the device current. The design of the PCB coil is similar for both the top and the bottom device. Therefore, for simplicity, only the design of coil for sensing current through $M_{2}$ is described.

Current from the source terminal of $M_{2}$ is routed through $T_{2}$ on the bottom layer of the half-bridge PCB as shown in Fig. 2(c). The PCB coil is fabricated above $T_{2}$ with traces on the second and third layer of the half-bridge PCB. The traces are joined with blind vias to connect the turns forming the PCB coil as shown in Fig. 2(d). The coil is, therefore, completely embedded within the half-bridge PCB. Further, the coil is placed such that its width overlaps trace $T_{2}$. In this way, the separation $\left(t_{3}\right)$ between $T_{2}$ and the PCB coil is minimized. This separation is set by the PCB stack-up specification at $0.35 \mathrm{~mm}$, which is significantly less than the minimum separation of $1.2 \mathrm{~mm}$ for the coil designed in [22]. Minimizing the separation between the PCB coil and $T_{2}$ increases the flux linkage per turn of the PCB coil. The lateral inter-turn separation between the coil turns, shown as $d$ in Fig. 2(d), is limited by the PCB manufacturing technology. Consequently, the number of turns in the PCB coil, placed within the width $(w)$ of $T_{2}$, is practically limited by $d$.

Fig. 3 shows the FEM model of the half-bridge layout developed to determine the power-loop inductance due to $T_{1}, T_{2}$, and the mutual inductance between $T_{2}$ and the PCB coil. To simplify the power-loop modeling, the current through the MOSFETs and the switching node trace, which connects the source of $M_{1}$ and the drain of $M_{2}$, is emulated via a single top-layer trace $T_{C}$ as shown in Fig. 3. To complete the power-loop model, the DC bus capacitors shown in Fig. 3 are modeled as solid conductors for routing the current through DC busbars. In this manner, a continuous power-loop consisting of traces $T_{1}, T_{C}, T_{2}$, and the DC busbars is formed. A current is injected through the power-loop by a current port $(P)$ modeled inside the trace $T_{C}$. A magnetostatic FEM

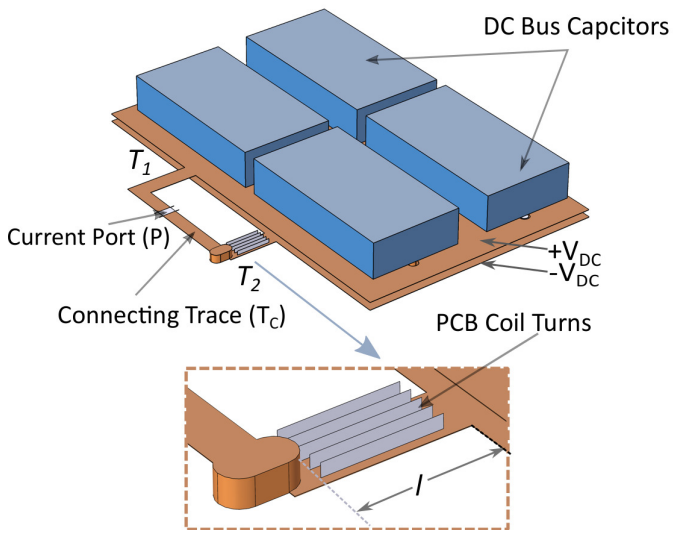

Fig. 3. FEM Model of the half-bridge layout shown in Fig. 2.

simulation is run to compute the magnetic field distribution $(B)$ in the region of permeability $\mu$ surrounding the model due to the current flow $\left(I_{M}\right)$ in the power-loop. The exterior boundary of this region, which spherically circumscribes the entire model shown in Fig. 3, is adjusted such that the magnetic field at the boundary has decayed substantially from its value near the power-loop. The power-loop inductance $(L)$ is then estimated as

$$
L=2\left(\int_{V} \frac{B^{2}}{2 \mu} d V\right) / I_{M}^{2}
$$

Furthermore, the magnetic field distribution $(B)$ is used to compute the mutual inductance $(M)$ between the trace $T_{2}$ and the PCB coil as

$$
M=\frac{\lambda}{I_{M}}
$$

where $\lambda$ is the total flux linking the PCB coil. $\lambda$ is computed through numerical integration of $B$ over the surface area outlined by the traces forming the PCB coil. Each turn of the PCB coil is represented through an array of planar surfaces as shown in Fig. 3. The elevation $\left(t_{3}\right)$ of these surfaces above $T_{2}$, their lateral separation $(d)$ and the height $\left(t_{2}\right)$ are set according to the dimensions of the coil shown in Fig. 2(d). The length of 


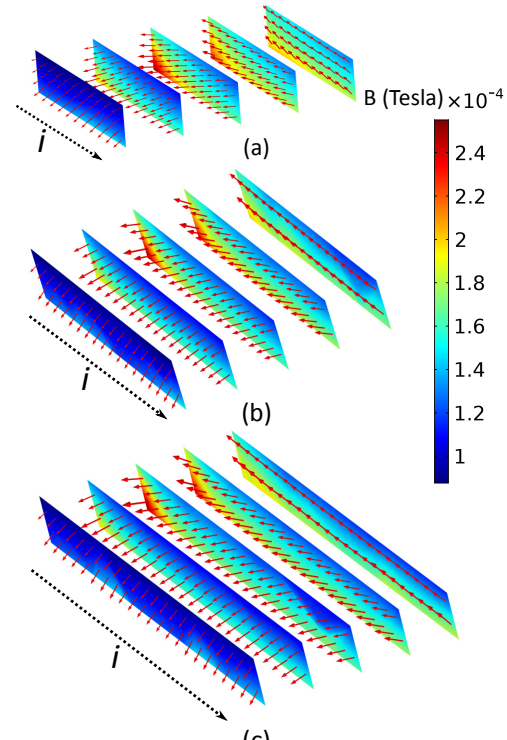

(c)

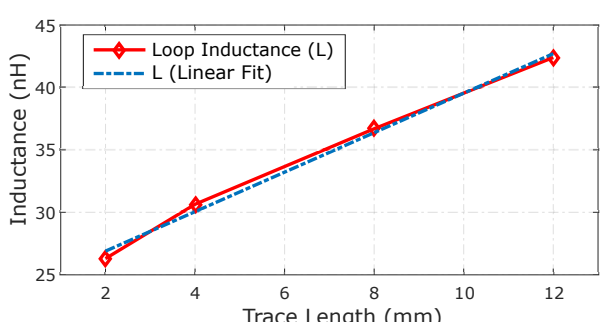

(a)

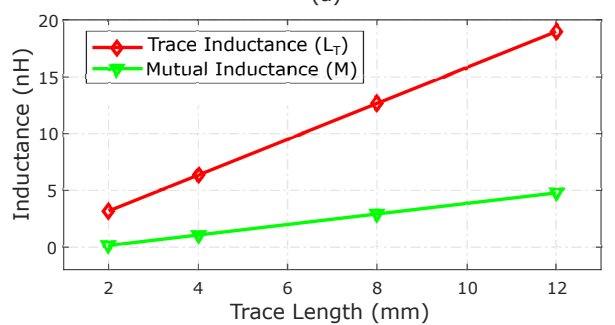

(b)
Fig. 4. Magnetic field distribution through the PCB coil with $I_{M}=1$ A for (a) $l=4 \mathrm{~mm}$ (b) $l=8 \mathrm{~mm}$, and (c) $l=12 \mathrm{~mm}$. Direction of magnetic field through the coil turns is indicated through arrows. Direction of current through the trace beneath the turns is also shown.

these turns $\left(l_{C}\right)$ is adjusted to account for all the flux linkages through the PCB coil.

It can be observed that increasing the length $(l)$ of $T_{2}$ can facilitate a coil design with larger turn area. A larger coil will have a higher mutual inductance with $T_{2}$ and hence higher sensitivity as well. However, increasing $l$ also increases the inductance of the power-loop, which is detrimental towards high speed operation of SiC MOSFETs. The power-loop inductance is estimated for $l=2 \mathrm{~mm}, 4 \mathrm{~mm}, 8 \mathrm{~mm}$, and $12 \mathrm{~mm}$ using (1). Both $T_{1}$ as well as $T_{2}$ are adjusted simultaneously to these lengths in the FEM model. Corresponding to each length the mutual inductance is also estimated using (2).

For $l=2 \mathrm{~mm}$, coil placement is not feasible due to inadequate region available above $T_{2}$ for fabricating the coil. For $l=4 \mathrm{~mm}, 8 \mathrm{~mm}$, and $12 \mathrm{~mm}$, magnetic field distribution $(B)$ through each turn of the PCB coil is shown in Fig. 4 for $I_{M}=1 \mathrm{~A}$. For all the trace lengths, power-loop inductance $(L)$ and the mutual-inductance $(M)$ between the coil and $T_{2}$ is plotted in Fig. 5(a) and Fig. 5(b), respectively. $L$ exhibits a slope of $1.58 \mathrm{nH} / \mathrm{mm}$ based on the linear fit through the data shown in Fig. 5(a). Since increase in $L$ is purely due to the increased length of traces $T_{2}$ and $T_{2}$, the trace inductance $\left(L_{T}=1.58 l\right)$ is estimated from this slope as shown in Fig. 5(b). $L_{T}$ includes the stray inductance contributed by both $T_{1}$ as well as $T_{2}$.

To minimize the power-loop inductance for designing a coil with any desired $M$, the minimum trace length required can be selected from Fig. 5(b). For the prototype presented in this article, the trace length is set at $8 \mathrm{~mm}$, which gives a mutual inductance of $2.9668 \mathrm{nH}$ between the trace and the PCB coil. Methodology for processing the designed PCB coil output for implementation of the ultrafast protection scheme is discussed in the next section.
Fig. 5. (a) Variation of power-loop inductance $(L)$ with trace length $l$. (b) Variation of mutual inductance $(M)$ between the PCB coil and $T_{2}$ and trace inductance $\left(L_{T}\right)$ with trace length $l$.

\section{Ultrafast Protection Circuit Design}

The gate driver circuit featuring ultrafast protection is shown in Fig. 6(a). The circuit is fabricated as a PCB card, shown in Fig. 6(b). Once connected to the main half-bridge board, the gate driver card receives the PCB coil output for further processing. The voltage induced by the device current $\left(I_{M}\right)$ across the PCB coil is expressed as

$$
V_{C}(t)=M \frac{d I_{M}(t)}{d t}
$$

The coil output voltage $\left(V_{C}\right)$ is integrated on the gate driver card to produce a voltage $\left(V_{S}\right)$, which is proportional to $I_{M}$. Integration is implemented using an analog integrator circuit configured in a non-inverting topology [23], as shown in Fig. 6(a). A high speed comparator compares $V_{S}$ with a fault reference voltage $V_{R E F}$. As will be discussed subsequently, $V_{R E F}$ can be set corresponding to any desired fault current level $I_{R E F}$. Once the fault is detected, the comparator output goes high at the instant $t_{D}$ shown in Fig. 6(c). This output is then latched and inverted before feeding it to the driver IC for disabling its output $\left(V_{G S}\right)$, which occurs at the instant $t_{P}$ shown in Fig. 6(c).

Having discussed the design of the PCB coil in the preceding section, the next step for implementation of the protection scheme is the design of the integrator and the logic circuit shown in Fig. 6(a). In this section, firstly, an expression for the current sensor frequency response is developed, which establishes the requisite high bandwidth current sensing capability of the PCB coil based current sensor. Thereafter, fault level adjustment for the protection circuit is discussed.

\section{A. Current Sensor Design}

The current sensor, comprising the PCB coil as well as the integrator circuit, must have sufficient bandwidth for tracking switching current through a SiC MOSFET. The upper 


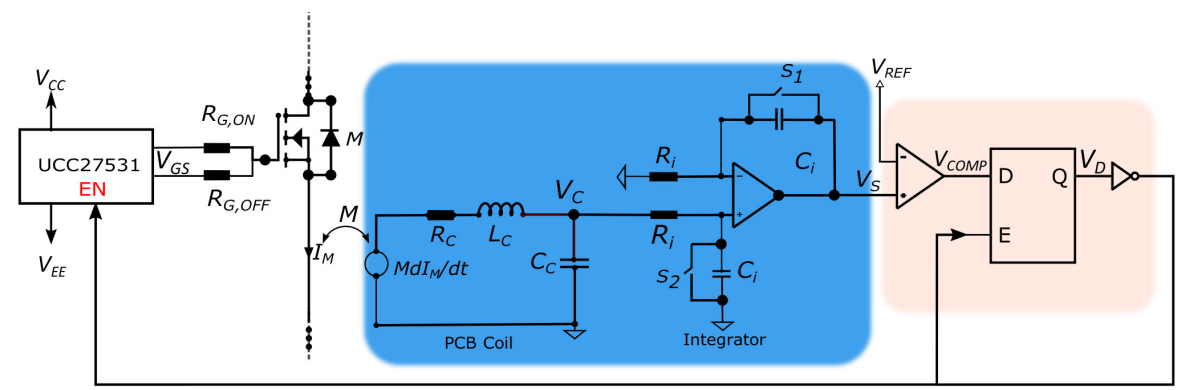

(a)
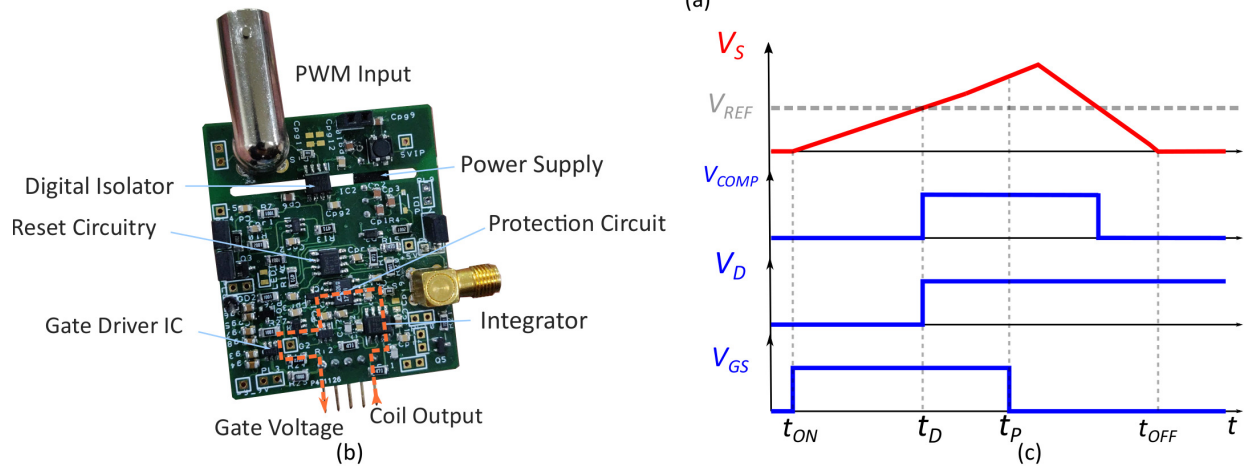

Fig. 6. (a) Gate driver schematic featuring the integrator and the protection circuitry. (b) PCB card implementing the gate driver schematic. (c) Simplified waveforms depicting the functioning of protection circuitry.

bandwidth limit (BW) [21] required to sense device current with a rise time $t_{r}(\mathrm{~ns})$ is

$$
B W(\mathrm{MHz})=\frac{0.35}{t_{r}} .
$$

For commercially available SiC MOSFETs, $d i / d t$ as high as $5.79 \mathrm{~A} / \mathrm{ns}$ is reported [24]. Therefore, the MOSFET current can rise quickly in the event of a short-circuit. From (4), sensing the device current with a typical rise time of 10 ns requires a current sensor bandwidth above $35 \mathrm{MHz}$. To determine the upper bandwidth limit of the designed PCB coil based current sensor, the parameters in the lumped model of the PCB coil, shown in Fig. 6(a), are first extracted using a 3D FEM simulation. A variable frequency current signal is injected through the terminals in the 3D model of the designed PCB coil shown in Fig. 2(d), and the corresponding terminal voltage is computed. With the terminal current and voltage known, the impedance at the coil terminals is computed. The reactive part of that impedance is plotted in Fig. 7. The reactive impedance in the low frequency region, shown zoomed in Fig. 7 , is used to estimate the lumped inductance $\left(L_{C}\right)$ of the PCB coil. Further, from the coil resonant frequency $\left(f_{r}\right)$, the coil lumped capacitance $\left(C_{C}\right)$ is estimated as

$$
C_{C}=\frac{1}{\left(2 \pi f_{r}\right)^{2} L_{C}} .
$$

The extracted parameters of the PCB coil are listed in Table. I. With these parameters, the transfer function for the coil $(C(s))$ is expressed as

$$
C(s)=\frac{V_{C}(s)}{I_{M}(s)}=\frac{M s\left(1+R_{i} C_{i} s\right)}{Z(s)}
$$

where,

$$
\begin{gathered}
Z(s)=R_{i} C_{i} L_{c} C_{c} s^{3}+\left(R_{c} C_{c} R_{i} C_{i}+L_{c} C_{i}+L_{c} C_{c}\right) s^{2} \\
+\left(R_{i} C_{i}+R_{c} C_{c}+R_{c} C_{i}\right) s+1 .
\end{gathered}
$$

A practical one-pole integrator model $\left(I_{P}(s)\right)$ [25], using an op-amp with finite open-loop gain $\left(a_{0}\right)$ and a finite unity gain-bandwidth $\left(\omega_{t}\right)$, is expressed as

$$
I_{P}(s)=\frac{V_{S}(s)}{V_{C}(s)}=\frac{a_{0}}{\left(1+\frac{s}{\omega_{0} / a_{0}}\right)\left(1+\frac{s}{\omega_{t}}\right)}
$$

where,

$$
\omega_{0}=\frac{1}{R_{i} C_{i}}
$$

is the unity gain frequency. From (6) and (8), the sensor transfer function $(T(s))$ can be expressed as

$$
T(s)=I_{P}(s) C(s)=\frac{a_{0} M s\left(1+R_{i} C_{i} s\right)}{Z(s)\left(1+\frac{s}{\omega_{0} / a_{0}}\right)\left(1+\frac{s}{\omega_{t}}\right)} .
$$

Using (10), the sensor frequency response $(T(j \omega))$ is plotted for $R_{i}=470 \Omega$ and $C_{i}=0.1 \mathrm{nF}$ as shown in Fig. 8(a). $T(j \omega)$ is plotted for a commercially available op-amp (THS4631) with $a_{0}$ and $f_{t}$ as $80 \mathrm{~dB}$ and $325 \mathrm{MHz}$, respectively. $T(j \omega)$ is also plotted through an LTspice simulation using manufacturer provided spice model of THS4631 in Fig. 8(b). The frequency response in Fig. 8(b) exhibits a larger gain roll-off at high frequency, which can be attributed to higher-order poles included in the manufacturer spice model. Irrespective of the roll-off at high frequencies, it is observed that the measurement requirement of $35 \mathrm{MHz}$ for a rise time of $10 \mathrm{~ns}$ is well within the flat response region of the frequency response. 


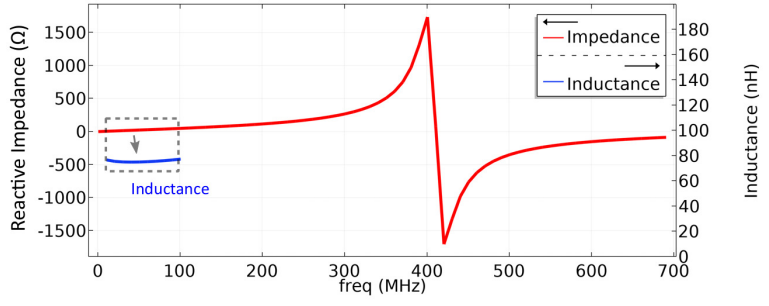

Fig. 7. Frequency response of the PCB coil.

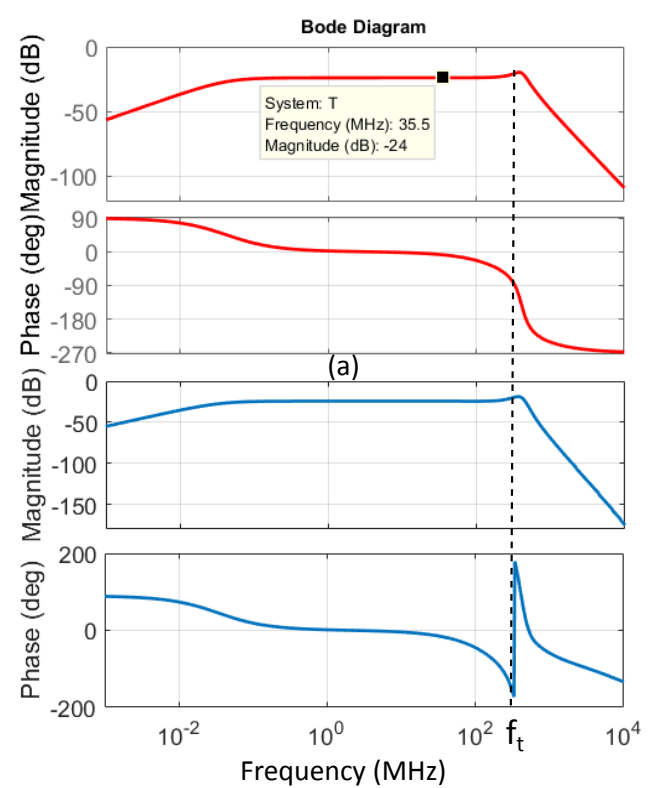

(b)

Fig. 8. Current sensor frequency response. (a) Sensor frequency response using the derived model $(T(j \omega))$. (b) Sensor frequency response generated through LTspice simulation.

TABLE I

CoIl PARAMETERS.

\begin{tabular}{ll}
\hline \hline Element & Magnitude \\
\hline Inductance $\left(L_{C}\right)$ & $73.24 \mathrm{nH}$ \\
Resistance $\left(R_{C}\right)$ & $0.314 \Omega$ \\
Capacitance $\left(C_{C}\right)$ & $2.043 \mathrm{pF}$ \\
Mutual inductance $(M)$ & $2.9668 \mathrm{nH}$ \\
Coil resonant frequency $\left(f_{r}\right)$ & $411.43 \mathrm{MHz}$ \\
\hline \hline
\end{tabular}

\section{B. Fault Level Adjustment}

After ignoring the coil parasitic inductance and capacitance, the sensor output voltage $\left(V_{S}\right)$ is simply expressed as

$$
V_{S}(t)=\frac{M i_{M}(t)}{R_{i} C_{i}} .
$$

Using the expression in (11) for adjustment of $V_{R E F}$ is justified since the frequency response $(T(j \omega))$ of the sensor ensures adequate bandwidth to track switching current transitions through the MOSFET. Therefore, the reference voltage $\left(V_{R E F}\right)$ for the comparator shown in Fig. 6(a), corresponding to fault current $\left(I_{R E F}\right)$, is adjusted as

$$
V_{R E F}=\frac{M I_{R E F}}{R_{i} C_{i}} .
$$

To avoid false triggering of the protection circuit, the error in the sensor output due to non-ideal circuit components can be estimated beforehand. Generally, the error $(d z)$ in $z=f\left(x_{1}, x_{2}, \ldots x_{n}\right)$ due to uncertainty in $x_{1}, x_{2}, \ldots x_{n}$ is expressed as

$$
d z=\sqrt{\left(\frac{\partial F}{\partial x_{1}}\right)^{2} d x_{1}^{2}+\left(\frac{\partial F}{\partial x_{2}}\right)^{2} d x_{2}^{2}+\cdots\left(\frac{\partial F}{\partial x_{n}}\right)^{2} d x_{n}^{2}}
$$

Using (13), the error in the sensor output (11) due to uncertainty in the value of $R_{i}, C_{i}$ is expressed as

$$
\frac{d V_{S}}{V_{S}}=\sqrt{\left(\frac{d R_{i}}{R_{i}}\right)^{2}+\left(\frac{d C_{i}}{C_{i}}\right)^{2}} .
$$

Since the component datasheets specify the uncertainty values, the expression in (14) can be used to set $V_{R E F}$ while accommodating any error in the sensor output. Another source of error for the integrator circuit is the op-amp input offset voltage $\left(V_{O S}\right)$ [23]. Besides the expression in (11), the sensor output also contains an error term due to integration of $V_{O S}$. The sensor output including the error from $V_{O S}$ is expressed as

$$
V_{S}(t)=\frac{M i(t)}{R_{i} C_{i}}+\frac{V_{O S} t}{R_{i} C_{i}}
$$

where $t$ is the duration from the instant when the power device is turned on. The error increases once the device starts conducting and reaches its maximum value just before the device is turned off. The error is reset to zero once the device is turned-off using switches $S_{1}$ and $S_{2}$ shown in Fig. 6(a). This periodic reset helps prevent the accumulation of error in the sensor output, which may otherwise saturate the integrator op-amp. Therefore, the error in the sensor output due to opamp input offset voltage during HSF or FUL is negligible, as the fault occurs just after the device is turned on and no significant accumulation of error has occurred. However, the error can be significant during high inductance faults, where the fault current rises slowly after the device is turned on. As such, $V_{R E F}$ can be adjusted appropriately based on $V_{O S}$ of the op-amp and the inductance of the circuit.

\section{Experimental Results}

The developed half-bridge prototype is shown in Fig. 9. The half-bridge circuit employs two UnitedSiC SiC devices (UF3C120080K4S). The gate driver card, shown in Fig. 6(b), is shown connected to the half-bridge board in Fig. 9. The driver IC (UCC27531) used in the driver card is a simple nonisolated IC that provides sufficient peak current for driving a discrete $\mathrm{SiC}$ MOSFET. Isolation from the control ground is provided through a digital isolator (ISO7710) having high common-mode transient immunity. A high speed comparator (LT1719) is used in the protection circuit to minimize propagation delay. Further, all the components on the driver card 


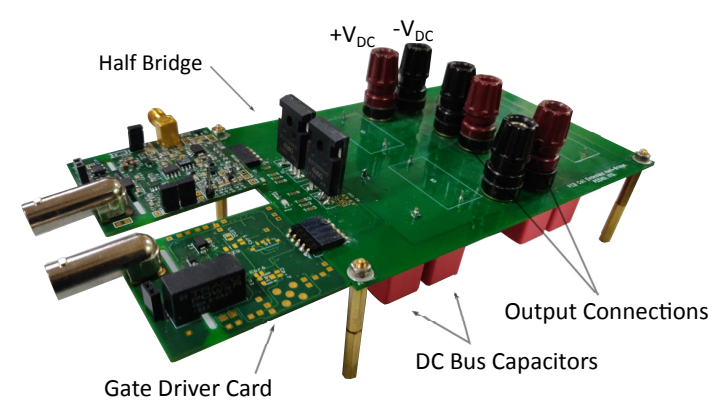

Fig. 9. Developed half-bridge prototype. The gate driver card is shown externally connected to the half-bridge board.

are powered by a single $2 \mathrm{~W}$ power supply (TBA 2-0522). Both the integrator op-amp (THS4631) and the comparator (LT1719) are powered with a bipolar voltage supply $( \pm 5 \mathrm{~V})$ generated on the driver card from the output of TBA 2-0522.

In this section, firstly, results from the experiments performed on the current sensor are presented. Secondly, results from the experiments performed on the protection circuit are presented. These results demonstrate the ultrafast response speed of the protection scheme. Results from both HSF as well as FUL tests on the SiC MOSFET are presented.

\section{A. Current Sensor Evaluation}

A $580 \mu \mathrm{H}$ inductor is connected between $D C+$ and $S W$ terminals shown in Fig. 2(a). Further, MOSFET $M_{2}$ is driven by four consecutive gating pulses, which results in four current pulses through the device. A TRCP0030 probe from Tektronix is used to evaluate the performance of the designed current sensor when measuring these current pulses, as shown in Fig. 10 . Both the sensor measurement $\left(V_{S}\right)$ and the Tektronix probe measurement $\left(I_{M}\right)$ are shown in Fig. 10. From Fig. 10, it is observed that the sensor can track the rising and falling current transitions through the SiC MOSFET with the required bandwidth.

The mutual inductance between the trace $T_{2}$ and the PCB coil can be estimated from Fig. 10(b) at the instant when the device current reaches its steady-state after it is turned on. Device current of $15 \mathrm{~A}$ is seen to produce a sensor output voltage of $1 \mathrm{~V}$. Consequently, for $R_{i}=470 \Omega$ and $C_{i}=0.1$ $\mathrm{nF}, M$ is estimated to be $3.13 \mathrm{nH}$ using (11), which is near the value $(2.9668 \mathrm{nH})$ estimated from FEM simulations in section II. This experimentally determined value of $M$ is utilized for adjusting the reference fault level of the protection circuit.

The sensor output exhibits an offset, due to op-amp input offset voltage, before the application of pulses to the MOSFET gate, as seen in Fig. 10(a). The integrator circuit capacitors are reset by switches $S_{1}$ and $S_{2}$ shown in Fig. 6(a) after the first gate pulse ends and the device is turned off. Thereafter, by periodically resetting the capacitors after the device is turned off, the accumulation of error in the sensor output is prevented.

\section{B. Hard Switched Fault}

Fig. 11 shows the half-bridge circuit as well as the gating pulse logic for conducting SC tests on the DUT, $M_{2}$. For

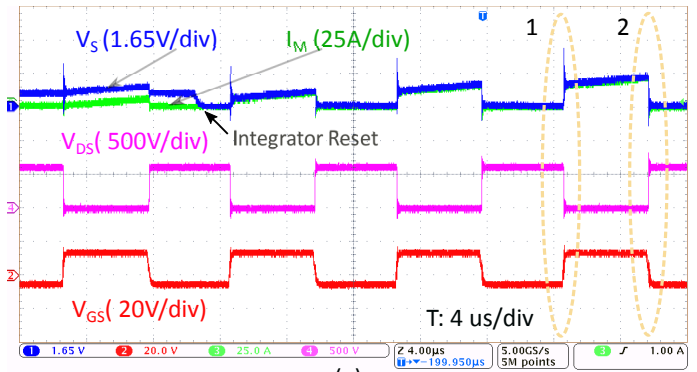

(a)

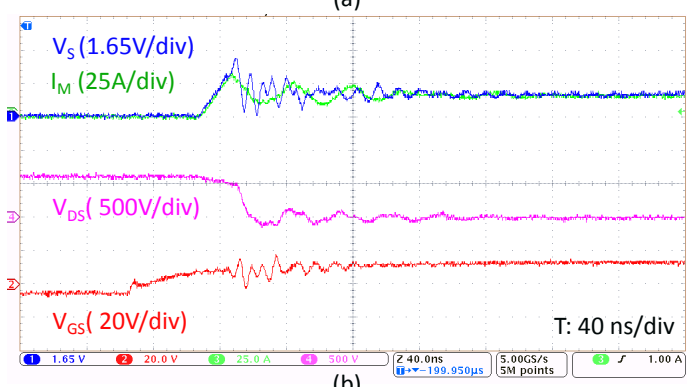

(b)

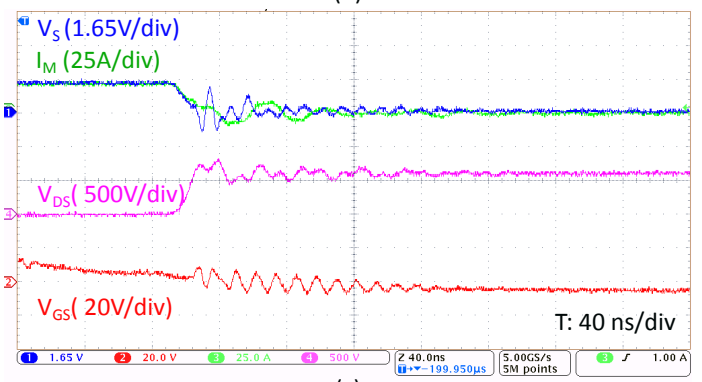

(c)

Fig. 10. (a) Pulse test results showing the device current measurement using the designed current sensor as well as a commercial probe. (b) Turn-on transition waveforms at the instant marked 1 in (a). (c) Turn-off transition waveforms at the instant marked 2 in (a).

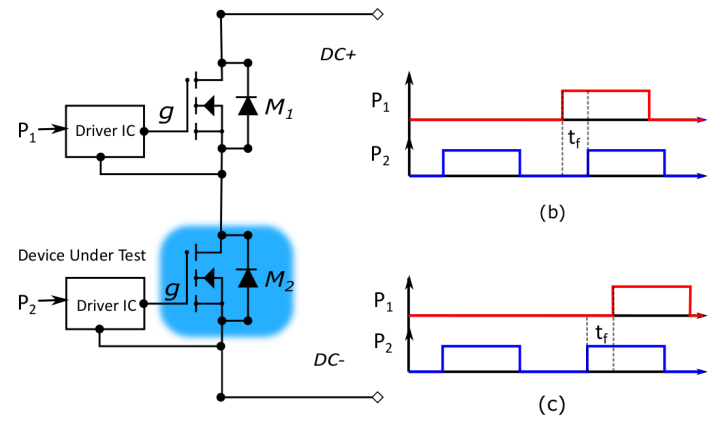

(a)

Fig. 11. (a) Half-bridge test setup showing the device under test (DUT) $M_{2}$. (b) Gate logic for conducting HSF. (c) Gate logic for conducting FUL.

conducting HSF, $M_{1}$ is first turned on by issuing a single gate pulse and $t_{f}$ interval afterwards, $M_{2}$ is switched on as shown in Fig. 11(b). For conducting FUL, $M_{2}$ is turned on first. After an interval $t_{f}, M_{1}$ is turned on. Furthermore, a continuous pulse train is fed to $M_{2}$ while a single fault creating pulse $P_{1}$ is fed to $M_{1}$. Continuous pulse train to $M_{2}$ ensures that the integrator capacitors are being reset periodically.

Measurements for the sensor voltage $\left(V_{S}\right)$ and the device current $\left(I_{M}\right)$, when $M_{2}$ is hard switched into a fault state, are shown in Fig. 12. $V_{D S}$ transitions from 0 to $600 \mathrm{~V}$, for 


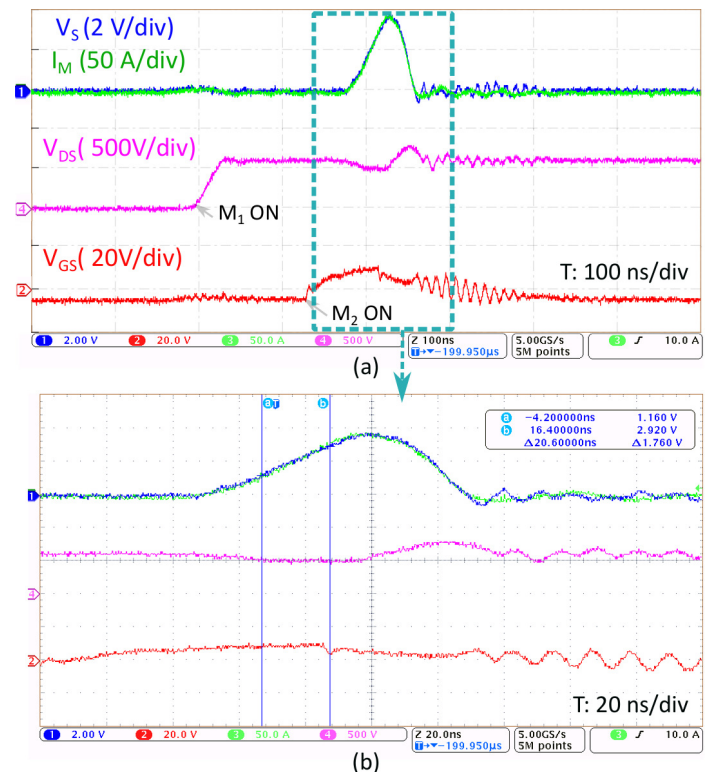

(b)

Fig. 12. (a) Experimental results demonstrating the protection of the device $M_{2}$ subjected to a HSF. (b) The indicated zoom region in (a) showing the approximate instant at which the protection circuit responds after detecting the fault.

$M_{2}$, when $M_{1}$ is turned on as shown in Fig. 12(a). After some duration, $M_{2}$ is turned on, hence short-circuiting the half-bridge leg. The protection circuit acts almost immediately to withdraw the gate pulse to $M_{2}$ after the fault reference level $\left(V_{R E F}\right)$ is detected by the protection circuit.

When $V_{R E F}$ is set at $1.1 \mathrm{~V}$, the current reference $\left(I_{R E F}\right)$ for $R_{i}=806 \Omega$ and $C_{i}=0.1 \mathrm{nF}$ is

$$
I_{R E F}=\left.\left(\frac{R C}{M}\right) V_{R E F}\right|_{V_{R E F}=1.1 \mathrm{v}} \approx 28 \mathrm{~A} .
$$

After the fault is detected, the gate driver circuit withdraws gate pulse to $M_{2}$ within 25 ns, as seen in Fig. 12(b). Therefore, the protection scheme demonstrates an ultrafast response time.

\section{Fault Under Load}

As depicted in Fig. 11(c), FUL test is carried out by first turning on $M_{2}$ and then subsequently turning on $M_{1}$. Fig. 13 shows the measurement results for the sensor voltage $\left(V_{S}\right)$ and the device current $\left(I_{M}\right)$ when $M_{2}$ is subjected to a FUL. For this experiment, the integrator components are set as: $R_{i}=2 \mathrm{k} \Omega$ and $C_{i}=0.1 \mathrm{nF}$. The current reference $I_{R E F}$ corresponding to these component values and when $V_{R E F}$ is adjusted to $500 \mathrm{mV}$ is

$$
I_{R E F}=\left.\left(\frac{R C}{M}\right) V_{R E F}\right|_{V_{R E F}=500 \mathrm{mV}} \approx 32 \mathrm{~A} .
$$

The driver IC (UCC27531) withdraws gate pulses to $M_{1}$ within $25 \mathrm{~ns}$ after detection of the fault, as can be observed from Fig. 13(b). Due to the turn-off delay time of $M_{2}$, the device current increases to a peak value of around $170 \mathrm{~A}$ after the fault is detected. Consequently, a low value of turnoff gate resistance is required to swiftly turn off the device without significant current rise through the device in the

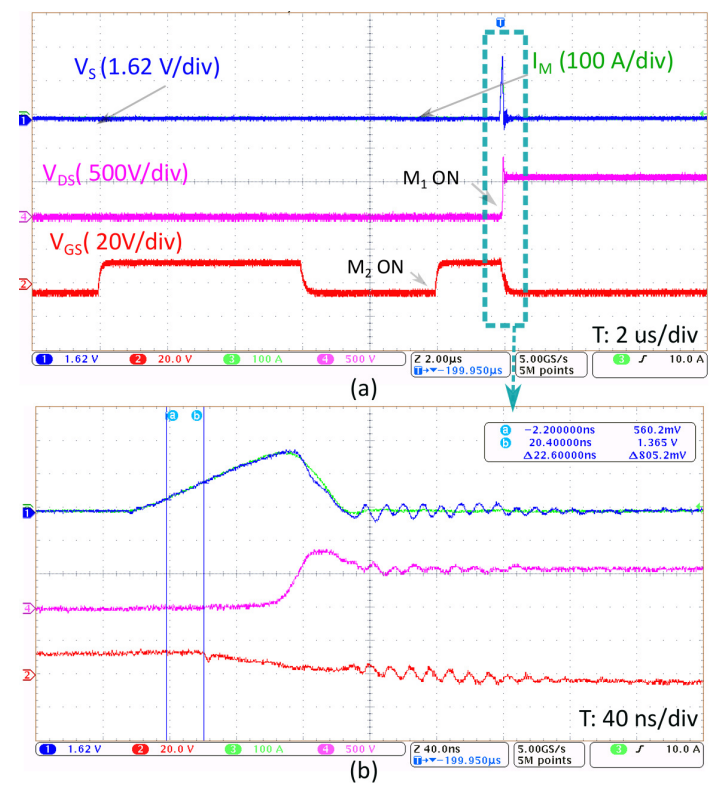

Fig. 13. (a) Experimental results demonstrating the protection of the device $M_{2}$ subjected to a FUL. (b) The indicated zoom region in (a) showing the approximate instant at which the protection circuit responds after detecting the fault.

event of a short-circuit. However, swiftly turning off $M_{2}$ may cause significant voltage stress across the device, which may cause device failure. As such, some gate drivers employ a soft turn-off technique to address this issue. The overvoltage experienced by the device, with a turn-off gate resistance of $20 \Omega$, is well below the maximum device rating of $1.2 \mathrm{kV}$, as observed in Fig. 13.

\section{CONCLUSION}

The application of PCB coils for implementing a protection scheme for discrete $\mathrm{SiC}$ MOSFETs was discussed in this article. Experimental results demonstrate ultrafast response time of the protection circuitry. Due to the flexibility of designing PCB coils on the power board itself, there is only the requirement of augmenting the gate driver circuit with additional protection circuitry. Moreover, the availability of the device current signal on the gate driver board can facilitate control and condition monitoring techniques for the power device.

\section{REFERENCES}

[1] J. Biela, M. Schweizer, S. Waffler, and J. W. Kolar, "Sic versus si evaluation of potentials for performance improvement of inverter and dc dc converter systems by sic power semiconductors," IEEE Trans. Ind. Electron., vol. 58, no. 7, pp. 2872-2882, 2011.

[2] J. Rabkowski, D. Peftitsis, and H.-P. Nee, "Silicon carbide power transistors: A new era in power electronics is initiated," IEEE Ind. Electron. Mag., vol. 6, no. 2, pp. 17-26, 2012.

[3] L. Ceccarelli, P. Reigosa, F. Iannuzzo, and F. Blaabjerg, "A survey of sic power mosfets short-circuit robustness and failure mode analysis," Microelectron. Reliab., vol. 76-77, pp. 272-276, 2017.

[4] J. Rice and J. Mookken, "Sic mosfet gate drive design considerations," in 2015 IEEE International Workshop on Integrated Power Packaging (IWIPP), 2015, pp. 24-27.

[5] Z. Wang, X. Shi, Y. Xue, L. M. Tolbert, F. Wang, and B. J. Blalock, "Design and performance evaluation of overcurrent protection schemes for silicon carbide (sic) power mosfets," IEEE Trans. Ind. Electron., vol. 61 , no. 10 , pp. 5570-5581, 2014. 
[6] B. Kakarla, T. Ziemann, R. Stark, P. Natzke, and U. Grossner, "Short circuit ruggedness of new generation $1.2 \mathrm{kv}$ sic mosfets," in 2018 IEEE 6th Workshop on Wide Bandgap Power Devices and Applications (WiPDA), 2018, pp. 118-124.

[7] Z. Wang, X. Shi, L. M. Tolbert, F. Wang, Z. Liang, D. Costinett, and B. J. Blalock, "Temperature-dependent short-circuit capability of silicon carbide power mosfets," IEEE Trans. Power Electron., vol. 31, no. 2 , pp. 1555-1566, 2016.

[8] D. Sadik, J. Colmenares, G. Tolstoy, D. Peftitsis, M. Bakowski, J. Rabkowski, and H. Nee, "Short-circuit protection circuits for siliconcarbide power transistors," IEEE Trans. Ind. Electron., vol. 63, no. 4, pp. 1995-2004, 2016.

[9] D. O'Sullivan, "Igbt overcurrent and short-circuit protection in industrial motor drives." Analog Device, 2015.

[10] X. Zhang, H. Li, J. A. Brothers, L. Fu, M. Perales, J. Wu, and J. Wang, "A gate drive with power over fiber-based isolated power supply and comprehensive protection functions for 15-kv sic mosfet," IEEE Journal of Emerging and Selected Topics in Power Electronics, vol. 4, no. 3, pp. 946-955, 2016.

[11] Y. Shi, R. Xie, L. Wang, Y. Shi, and H. Li, "Switching characterization and short-circuit protection of $1200 \mathrm{v}$ sic mosfet t-type module in pv inverter application," IEEE Trans. Ind. Electron., vol. 64, no. 11, pp. 9135-9143, 2017.

[12] S. Ji, M. Laitinen, X. Huang, J. Sun, W. Giewont, F. Wang, and L. M. Tolbert, "Short-circuit characterization and protection of 10-kv sic mosfet," IEEE Trans. Power Electron., vol. 34, no. 2, pp. 1755-1764, 2019.

[13] "C3m0075120k, silicon carbide power MOSFET, c3m MOSFET technology.” Tech. Rep. [Online]. Available: https://www.mouser.ec/ datasheet/2/90/c3m0075120k-2325780.pdf

[14] K. Sun, J. Wang, R. Burgos, and D. Boroyevich, "Design, analysis, and discussion of short circuit and overload gate-driver dual-protection scheme for 1.2-kv, 400-a sic mosfet modules," IEEE Trans. Power Electron., vol. 35, no. 3, pp. 3054-3068, 2020.

[15] J. Xue, Z. Xin, H. Wang, P. C. Loh, and F. Blaabjerg, "An improved di/dt-rcd detection for short-circuit protection of sic mosfet," IEEE Trans. Power Electron., vol. 36, no. 1, pp. 12-17, 2021.

[16] D. Rothmund, D. Bortis, and J. W. Kolar, "Highly compact isolated gate driver with ultrafast overcurrent protection for $10 \mathrm{kv}$ sic mosfets," CPSS Transactions on Power Electronics and Applications, vol. 3, no. 4, pp. 278-291, 2018.

[17] J. Wang, S. Mocevic, R. Burgos, and D. Boroyevich, "High-scalability enhanced gate drivers for sic mosfet modules with transient immunity beyond 100Â v/ns," IEEE Trans. Power Electron., vol. 35, no. 10, pp. $10180-10199,2020$.

[18] D. Gerber, T. Guillod, R. Leutwyler, and J. Biela, "Gate unit with improved short-circuit detection and turn-off capability for $4.5-\mathrm{kv}$ presspack IGBTs operated at 4-ka pulse current," IEEE Trans. Plasma Sci. vol. 41, no. 10, pp. 2641-2648, Oct 2013.

[19] S. Mocevic, J. Wang, R. Burgos, D. Boroyevich, M. Jaksic, C. Stancu, and B. Peaslee, "Comparison and discussion on shortcircuit protections for silicon-carbide mosfet modules: Desaturation versus rogowski switch-current sensor," IEEE Trans. Ind. Appl., vol. 56, no. 3, pp. 2880 2893, 2020.

[20] Y. Kuwabara, K. Wada, J. Guichon, J. Schanen, and J. Roudet, "Implementation and performance of a current sensor for a laminated bus bar," IEEE Trans. Ind. Appl., vol. 54, no. 3, pp. 2579-2587, May 2018.

[21] K. Wang, X. Yang, H. Li, L. Wang, and P. Jain, "A high-bandwidth integrated current measurement for detecting switching current of fast GaN devices," IEEE Trans. Power Electron., vol. 33, no. 7, pp. 61996210, July 2018.

[22] A. Rafiq, S. Pramanick, and R. Maheshwari, "Design of pcb coil based high bandwidth current sensor with power-loop stray inductance characterization," IEEE Trans. Ind. Electron., pp. 1-1, 2020.

[23] A. Radun, "An alternative low-cost current-sensing scheme for highcurrent power electronics circuits," IEEE Trans. Ind. Electron., vol. 42, no. 1, pp. 78-84, Feb 1995.

[24] "Test report: Driving cree c3m SiC MOSFETs with silicon labs si828x gate drivers in applications requiring shortcircuit protection," Cree, Tech. Rep. [Online]. Available: https://www.wolfspeed.com/downloads/dl/file/id/2001/product/643/ driving_cree_c3m_sic_mosfets_with_silicon_labs_si828x_gate_drivers_ in_applications_requiring_short_circuit_protection.pdf

[25] S. Franco, Design with Operational Amplifiers \& Analog Integrated Ciruits, 3rd ed. McGraw Hill. 